Notfall Rettungsmed 2016 · 19:323-324

DOI 10.1007/s10049-016-0191-4

Online publiziert: 24. Juni 2016

๑) Springer-Verlag Berlin Heidelberg 2016

CrossMark

Lieber Leserinnen, liebe Leser,

insbesondere im letzten Jahr hat das Eintreffen einer großen Zahl von Flüchtlingen das Erstversorgungssystem in Deutschland mit einer Fülle von neuen Aufgaben konfrontiert.

Es kam zu täglich wechselnden, teilweise kaum vorhersehbaren Flüchtlingszahlen, insbesondere über die Balkanroute mit der ersten medizinischen Versorgung in München (bis zu 20.000/ Wochenende). Viele dieser Flüchtlinge hatten eine lange und teilweise unter lebensbedrohlichen Bedingungen überstandene Fluchtroute hinter sich. Dieses unplanbare Auftreten einer erheblichen Anzahl von Personen, die teilweise medizinischer Versorgung bedurften, ist in beispielhafter Weise durch die Kommunalverwaltungen und das medizinische System in Deutschland bewältigt worden.

Aus dieser Erfahrung heraus haben sich diverse organisatorische und medizinisch-inhaltliche Lehren ergeben, die auch für die Regelversorgung oder für die Planungen bei Anfall hoher Patientenzahlen für die Zukunft lehrreich sind.

Im vorliegenden Heft ist es gelungen einige kompetente Autoren mit eigenen aktuellen Erfahrungen für die Darstellung dieser Themen zu gewinnen. Das Spektrum reicht von der juristischen und versicherungsrechtlichen Seite, wie sie von P. Klein dargestellt wird, bis zur Erkenntnis, dass eine integrierende Versorgung von Flüchtlingen insbesondere in der Erstkontaktsituation häufig nur durch die Inanspruchnahme von freiwilligen Helfern möglich ist. Hier werden im Artikel von I. Heer Beispiele dargestellt wie eine solche Einsatzrealität sowohl medizinisch sachgerecht wie auch juristisch korrekt organisiert werden kann.

\title{
T. Nicolai
}

Dr. von Haunersches Kinderspital, Kinderklinik und Kinderpoliklinik, Ludwig Maximilian Universität München, München, Deutschland

\section{Akutversorgung für Flüchtlinge}

Dabei zeigten sich die immense Hilfsbereitschaft des medizinischen Personals - von der Pflege bis zu den Ärzten - und die meist gute und einfallsreiche Organisation seitens der zuständigen Behörden.

Einen für alle Versorger relevanten Aspekt stellt auch die Frage dar, inwieweit mit besonderen Infektionskrankheiten $\mathrm{zu}$ rechnen ist und wie gegebenenfalls deren Ausbreitung innerhalb der Flüchtlingsgemeinschaft in den jeweiligen Massenunterkünften, aber auch in der allgemeinen Bevölkerung verhindert werden kann. Hierzu wird auch insbesondere das Thema der Erstuntersuchung und des Screenings durch C. Rauscher in diesem Themenheft ausführlich bearbeitet.

Es hat sich gezeigt, dass die Mehrzahl der Flüchtenden ähnliche Erkrankungsmuster wie die Wohnbevölkerung zeigt und keineswegs mit einer großen Anzahl hoch ansteckender oder tropischer Infektionskrankheiten zu rechnen ist. Trotzdem sind hier einige Besonderheiten zu beachten, z. B. traten insbesondere das Läuse-Rückfallfieber und ähnliche hier bisher wenig bekannte Erkrankungen bei Flüchtlingen auf und führten zu teilweise nur intensivmedizinisch zu beherrschenden septischen Verläufen. M. Hufnagel erläutert, wie hier ein sinnvolles Vorgehen aussehen kann.

Besonderes Geschick ist erforderlich, um eine große Zahl von Menschen mit ganz unterschiedlichen sprachlichen und kulturellen Hintergründen so zu versorgen, dass ihre Mitarbeit gesichert und ihr Vertrauen erworben werden kann, ohne das eine erfolgreiche medizinische Versorgung nicht möglich wäre. S. Pluntke stellt dieses Thema, das auch in der weiteren Betreuung dieser Patientengruppe eine große Rolle spielen wird, in die Tiefe gehend und praxisbezogen dar.
Man erkennt aus den hier berichteten Erfahrungen, dass die Ankunft vieler Familien, die teilweise außerordentlich schwere Schicksalsschläge erlebt hatten und nach häufig komplikationsreicher Flucht mit Entbehrungen, Verletzungen und psychischen Stressfaktoren in Deutschland ankamen, nicht nur gut bewältigt werden konnte, sondern dass dies auch für alle Beteiligten durch die durchweg große Dankbarkeit seitens der Flüchtlinge zu einem bereichernden Erlebnis werden konnte. Die Wahrnehmung der Flüchtlinge in ihrer Individualität und nicht als Masse ist hierbei ein entscheidendes Element, das sich aus der medizinischen Ethik von selbst ergibt. Aus dieser Haltung heraus haben sich auch viele kreative Lösungsansätze gefunden, wie sie in den hier vorgelegten Artikeln beschrieben werden. Probleme mit Kommunikation, kulturellen Differenzen und der Begrenzung der zur Verfügung stehenden Versorgungsmittel wurden dadurch lösbar.

\section{》) Eine erfolgreiche medizi- nische Versorgung ist nur mit Vertrauen möglich}

Wer selbst schon einmal in einer Notsituation auf fremde Hilfe angewiesen war oder gar in einem fremden Land ohne Sprachkenntnis in einer Notsituation zurechtkommen musste, wird die erfolgreiche und von den Flüchtlingen zumeist positiv wahrgenommene Versorgung durch das Medizinsystem mit Empathie und Dankbarkeit beobachtet haben.

Wir hoffen, dass die Darstellung dieser Erfahrungen die Bewältigung künftiger ähnlicher Situationen erleichtert. Es hat sich gezeigt, dass durch die Hilfs- 
bereitschaft der Bevölkerung sowie der versorgenden medizinischen Strukturen und des Fachpersonals auch sehr schwierige Aufgaben vorbildlich gelöst werden konnten.

Kritisches Thema der Zukunft dürfte die Betreuung von durch kriegsbedingten Stress und die Flucht traumatisierte Personen werden. Unbegleitete Jugendliche und Kinder stellen eine weitere Personengruppe dar, die einer besonderen Fürsorge bedürfen wird. Diese haben sich zum Teil einer Registrierung und Betreuung entzogen; auf sich allein gestellt sind sie besonders gefährdet. Hier sollte unsere zukünftige Aufmerksamkeit besonders fokussiert werden.

\section{ueser}

T. Nicolai

\section{Korrespondenzadresse}

\section{Prof. Dr. T. Nicolai}

Dr. von Haunersches Kinderspital, Kinderklinik und Kinderpoliklinik, Ludwig Maximilian Universität München

Lindwurmstr. 4, 80337 München, Deutschland Thomas.Nicolai@med.uni-muenchen.de

Interessenkonflikt. T. Nicolai gibt an, dass kein Interessenkonflikt besteht.

\section{Projekt OrphanAnesthesia}

Anästhesie-Handlungsempfehlungen für Patienten mit seltenen Erkrankungen

OrphanAnesthesia (www. orphananesthesia.eu) ist ein krankheitsübergreifendes wissenschaftliches Projekt der Deutschen Gesellschaft für Anästhesiologie und Intensivmedizin e.V. in Zusammenarbeit mit Orphanet sowie der Europäischen Gesellschaft für Kinderanästhesie. Das Projekt hat sich zur Aufgabe gemacht, Handlungsempfehlungen zur Anästhesie bei Patienten mit seltenen Erkrankungen zu erstellen. In der Europäischen Union gilt eine Erkrankung als selten, wenn nicht mehr als 5 von 10.000 Menschen in der EU von ihr betroffen sind. Patienten mit einer seltenen Erkrankung benötigen häufig eine Vielzahl von chirurgischen Eingriffen, sowohl zur primären Versorgung der Krankheit als auch zur Therapie von Erkrankungen, die nicht zwingend mit der Grunderkrankung in Verbindung stehen. Genau wie "gesunde" Menschen können auch diese Patienten Notfallsituationen erleiden, die eine sofortige Narkose erfordern. Bei der großen Anzahl von seltenen Erkrankungen ist damit zu rechnen, dass der behandelnde Narkosearzt nicht auf umfangreiche persönliche Erfahrung zurückgreifen kann und somit auf weitere Informationen angewiesen ist. Die Bedeutung der Anästhesie wird bei der Behandlung von Patienten mit seltenen Erkrankungen oft unterschätzt, da der Anästhesist natürlich mit der einzelnen Erkrankung oft nur wenig zu tun hat.

Die OrphanAnesthesia-Handlungsempfehlungen werden von einem Anästhesisten erstellt und nach einem Peer-Review-Verfahren (durch einen weiteren Anästhesisten und einen Krankheitsexperten, z.B. Pädiater, Neurologe) kostenfrei zugänglich gemacht. Der Peer-Review-Prozess sichert einen hohen Qualitätsstandard.

OrphanAnesthesia widmet sich nicht einer einzelnen Erkrankung, sondern möchte Informationen zu einer Vielzahl von seltenen Erkrankungen, bei denen mit einer Interaktion während der Narkose gerechnet werden muss, verfügbar machen. Die Vernetzung von Forschung sowie die enge Zusammenarbeit mit Patientenvereinigungen sind zentrale Anliegen von Orpha-
nAnesthesia. Das Projekt ist international ausgerichtet, wodurch die Handlungsempfehlungen grundsätzlich in englischer Sprache veröffentlicht werden. Bis zum jetzigen Zeitpunkt sind knapp 100 Handlungsempfehlungen publiziert, einige davon in bis zu 4 Sprachen.

Seit 2015 werden die Handlungsempfehlungen auch als Online-Supplement der Verbandszeitschrift Anästhesiologie \& Intensivmedizin (http://www.ai-online.info/ aionline/Orphsuppl) der DGAI veröffentlicht. Die Empfehlungen sind damit auch zitierfähig.

Ab September 2016 wird zusätzlich eine OrphanAnesthesia App das Angebot abrunden. Damit wird der Zugriff auf die Handlungsempfehlungen für Ärzte noch zeitgemäßer und unkomplizierter.

OrphanAnesthesia stellt somit einen sehr wichtigen Beitrag für die Patientensicherheit dar. Ein informiertes Team führt zu einer schnelleren und professionelleren Versorgung der oft durch mehrfache Krankenhausbesuche traumatisierten Patienten und deren Familien. Der Anästhesist kann das gesammelte Wissen für die Planung der Narkose nutzen und somit dem Patienten Sicherheit und die bestmöglichste Versorgung bieten.

Gerne können Sie sich am Projekt beteiligen! Wir freuen uns über Ihre Mitarbeit als Reviewer. Schlagen Sie uns auch gerne seltene Erkrankungen vor, die auf der Projektwebseite mit aufgenommen werden sollen.

Kontakt:

Prof. Dr. med. Tino Münster

Projektleitung OrphanAnesthesia

Universitätsklinikum Erlangen

Tel. 004991318533680

E-Mail

tino.muenster@kfa.imed.uni-erlangen.de 